\title{
Lamartine et les catholiques de France et du Canada (3ième partie et fin)
}

\section{Robert Sylvain}

Volume 4, numéro 3, décembre 1950

URI : https://id.erudit.org/iderudit/801654ar

DOI : https://doi.org/10.7202/801654ar

Aller au sommaire du numéro

Éditeur(s)

Institut d'histoire de l'Amérique française

ISSN

0035-2357 (imprimé)

1492-1383 (numérique)

Découvrir la revue

Citer cet article

Sylvain, R. (1950). Lamartine et les catholiques de France et du Canada (3ième partie et fin). Revue d'histoire de l'Amérique française, 4(3), 375-397.

https://doi.org/10.7202/801654ar d'utilisation que vous pouvez consulter en ligne.

https://apropos.erudit.org/fr/usagers/politique-dutilisation/ 


\title{
LAMARTINE ET LES CATHOLIQUES DE FRANCE ET DU CANADA
}

\author{
Bième partie et fin*
}

Les journaux canadiens, la Minerve et le Journal de Québec tout particulièrement, parce que les mieux informés de l'époque, avaient fait connaître à leurs lecteurs les efforts déployés pour intéresser l'opinion américaine au Cours familier de littérature. D'isolés qu'ils avaient été jusque-là, les articles et les communiqués concernant Lamartine se firent nombreux et suivis, lorsque Desplace arriva à Montréal, au début d'octobre 1856.

Mais la Minerve, dans la région montréalaise, ne tarda pas à se laisser devancer en ferveur lamartinienne par la Patrie, tandis que le Journal de Québec resta sans conteste le plus chaleureux panégyriste du poète dans la vieille capitale.

La raison de cette attitude était facile à déduire: ces deux journaux avaient pour principaux rédacteurs des Français. Alfred Rambeau et Émile Chevalier se partageaient la rédaction de la Patrie, tandis qu'un ancien collaborateur de l'Univers ${ }^{172}$, Émile de Fenouillet ${ }^{173}$, Écrivait au Journal de Québec.

Dès le 6 octobre, la Patrie publiait une lettre de Lamartine au maire de Montréal: "Je vous recommande, y lisait-on, aussi vivement

\footnotetext{
* Voir Revue d'Histoire de l'Amérique française (juin 1950): 29-61; (septembre 1950): 233-249.

172. Il s'en glorifiait dans une lettre à Joseph Doutre, 27 janvier 1856, inséré dans la Patrie du 22 février 1856. Cette assertion était fondée, comme il appert par différents numéros de l'Univers: 28 novembre 1851, 3 janvier, $17 \mathrm{mars}, 30 \mathrm{mai}$, ler juin et 1er juillet 1852 .

173. Il commença à collaborer au Journal de Québec, en octobre 1854, et remplaça Joseph Cauchon, comme rédacteur en chef, en janvier 1855 . Cf. Journal de Québec, 30 janvier 1855. Il se démit de sa fonction quand il fut nommé professeur à l'Ecole normale Laval, en février 1857. Cf. le même journal, 24 février 1857. - Le vrai nom de Fenouillet était Émile Régnier. Cf. C.-J. Magnan, Bulletin des recherches historiques, 47 (1941): 357-362.
} 
que j'en suis capable mon excellent ami M. Desplace, et l'affaire dont il a bien voulu se charger par pur dévouement à nos malheurs. Cette affaire contient mon salut et celui de huit cents pauvres paysans, compromis dans ma ruine, si cette ruine imméritée venait à s'accomplir faute de travail. En l'aidant, c'est moi que vous sauvez."

La Patrie continuait ses bons offices en annonçant, le 10 octobre, un banquet offert par les Français de Montréal à Desplace ${ }^{174}$. Le 24 octobre, le même journal communiquait à ses lecteurs les noms de ceux qui constituaient un comité d'aide à Lamartine. Cette liste était intéressante, car si elle mentionnait quelques membres en vue du parti conservateur, comme Pierre-Joseph-Olivier Chauveau, sous le patronage duquel se tenait ce comité, Thomas-Jean-Jacques Loranger et Louis-Victor Sicotte, elle énumérait également les noms des plus fougueux représentants du parti libéral-radical: celui de Louis-Joseph Papineau attirait tout de suite les regards, puis ceux de ses disciples à qui les violentes campagnes anticléricales du défunt Avenir, les thèses échevelées soutenues à la tribune de l'Institut Canadien de Montréal et une éclatante victoire remportée, malgré l'opposition du clergé, durant la campagne électorale de 1854, avaient conféré une renommée tapageuse:-Louis-Antoine Dessaulles, Charles Daoust, rédacteur au Pays, Antoine-Aimé Dorion, Joseph Doutre, Louis Labrèche-Viger, Robert Laflamme et Joseph Papin. Il n'y a pas de doute que bien des lecteurs de la Patrie ne se méprirent pas sur l'évolution ultérieure de la campagne d'abonnements au Cours familier. A une époque d'intenses rivalités politiques, qui dégénéraient vite, très souvent, en polémiques doctrinales, les moindres incidents donnaient lieu à des luttes exacerbées entre les deux partis: la souscription du poète ne pouvait échapper, de par la publicité dont elle était favorisée, à ce sort inéluctable.

Dès le 25 octobre, le Pays faisait mine de prendre la tête du mouvement au bénéfice du parti libéral-radical et de l'Institut Canadien c'était tout un -, dont il était le truchement autorisé. Son rédacteur écrivait, en effet:

Nous esperons que les membres de l'Institut s'empresseront de souscrire au Cours familier de littérature, car il leur

174. Alfred Rambeau y assistait. Sa dernière apparition en public avait donc eu lieu en faveur de son illustre compatriote, car il s'alitait le 17 octobre et décédait le 30 octobre. Cf. la Patrie, 31 octobre 1856. 
appartient, comme faisant partie de la première institution littéraire du Canada, de tendre la main au génie brillant et malheureux, et de prouver que la jeunesse du Canada sait apprécier autant que partout ailleurs l'homme qui, de tous les ecrivains contemporains, sait le mieux toucher le cœur et exciter l'admiration et l'enthousiasme de ses lecteurs.

Le même journal reproduisait ensuite l'Appel au Canada en faveur de Lamartine, déjà paru dans la Patrie du jour précédent, morceau dont les paragraphes courts, sobres, animés par endroits d'un pathétique discret, bien détachés les uns des autres, soulignaient en traits accentués, facilement visibles même aux plus myopes des lecteurs, la détresse du poète et le courage stoïque avec lequel il faisait face par le travail à la pauvreté menaçante. La rédaction de cette pièce se révélait habile: chaque phrase était calculée pour impressionner favorablement l'abonné éventuel du Cours familier. On n'oubliait pas la note religieuse, et ce n'est pas sans une surprise amusée que l'on dut lire cette prose dévote dans l'organe des anticléricaux de l'époque: "Un peuple pieux comme le nồtre n'a pas oublié les efforts de l'illustre poète pour arrêter son siècle sur la pente du matérialisme; il se souvient que Lamartine a puissamment contribué au retour des idées religieuses, en entretenant ses contemporains de l'âme et de Dieu."

Mis en veine, le Pays citait un extrait d'une lettre de Desplace, qui y allait, lui aussi, de son couplet édifiant: "Dans un pays comme le Canada, jaloux de conserver intacts ses principes religieux et la pureté des mœurs, le Cours familier de littérature se place naturellement sous le patronage des mères de famille. Le langage de Lamartine est aussi chaste que son style est beau. Il n'alarme jamais l'innocence. Non seulement il parle des choses sacrées avec le respect qui leur est dû, mais il l'inspire. Il enveloppe le lecteur d'une atmosphère bienfaisante qui élève l'âme et la fortifie."

Comme la clientèle du Pays se recrutait parmi les démocrates et les esprits "avancés", ce n'est pas parmi ses lecteurs - la lettre circulaire des Pères du second concile provincial de Québec, datée du 4 juin 1854, exhortait les fidèles à se détourner des mauvais journaux que poúvait surgir une mise au point pertinente touchant la religion de Lamartine. Elle vint d'abonnés à une feuille plus orthodoxe. Émile Chevalier, rédacteur à la Patrie, s'avisa d'y publier, du 27 octobre au 10 novembre, une notice biographique de Lamartine. Le 31 oc- 
tobre, il célébrait Jocelyn sur le mode lyrique: "Jocelyn, quel livre! Pour moi, je n'en ai jamais lu qui m'ait autant impressionné [ ... ]. Jocelyn, quel livre! je le répète, c'est l'écueil du scepticisme. Et il ment, ou il n'est pas homme celuí qui assure qu'il doute encore après avoir lu Jocelyn!"

Deux lecteurs de la Patrie, des Québécois, trouvèrent que cette fois l'on dépassait la mesure. D'autant plus que, à Québec, maintenant l'on battait la grosse caisse en faveur de Lamartine. "L'ami de M. Lamartine, M. Desplace, écrivait le rédacteur du Journal de Québec, le 30 octobre 1856, est arrivé enfin à Québec. Nous commencions à craindre que le bon accueil qui lui ont fait les Montréalais ne le retint chez eux jusqu'à la clôture de la navigation. Mais le voici au milieu de nous..." Et le journaliste montait sur le trépied pour célébrer à son tour le grand écrivain:

Lamartine, le plus magnifique poète de la langue française, l'illustration la plus pure de notre génération, le grand orateur, le seul tribun dont la parole ait pu enchaîner pendant trois mois toutes les passions populaires que la révolution de février avait soulevées, Lamartine est malheureux et, pour faire face à toutes les obligations qu'il a contractées, il ne pouvait trouver rien de mieux que d'ecrire encore quelques volumes. Ainsi, toutes les fois qu'elle est secouée par le vent de l'adversite, cette plume laisse tomber de nouvelles fleurs de poésie ${ }^{175}$, jonche le sol des fruits dorés de son éloquence; et les peuples bénissent presque le malheur qui force l'illustre barde à écrire sans cesse malgré ses vieux ans et toutes ses fatigues.

Un comité, dans lequel figuraient Octave Crémazie et FrançoisXavier Garneau, ne tardait pas à se constituer et les fervents des lettres se réunissaient, le 31 octobre, à l'hôtel de ville pour écouter Desplace discourir sur les vertus et les malheurs de son illustre ami.

Parmi ses auditeurs, il s'en trouva peut-être qui l'entendirent avec quelque malaise célébrer la piété de l'auteur de Jocelyn. Ayant déjà parcouru la Patrie du 27 octobre et reconnu, sous la plume du

175. Le journaliste se souvenait-il-de l'appréciation, par Gaillardet, de l'Histoire de César de Lamartine? Cette critique avait d'abord paru dans le Courrier des EtatsUnis, puis avait été reproduite par le Journal de Québec du 27 octobre 1855. On y lisait ces deux phrases: "Le talent est chez lui [ Lamartine ] un don de la nature. Il pousse comme les fruits sur les arbres, et il n'a qu'à secouer sa plume pour qu'il en tombe des fleurs de rhétorique." 
journaliste, des propos identiques, ils purent flairer l'origine d'un petit complot: une prompte rectification s'imposait donc, si l'on ne voulait pas qu'il y eût, dans le public qui lisait, un regrettable malentendu en une matière aussi importante que la signification exacte de la pensée et des œuvres d'un auteur acclamé par les cent bouches de la renommée.

Nous ne pouvons vous accorder, comme vous l'affirmez, écrivirent-ils à Chevalier, que Lamartine soit vraiment religieux, qu'il ait ces sentiments que vous lui attribuez. Lamartine est, sans doute, un grand poète et un grand littérateur; $c \theta-$ pendant le rôle qu'il a joué dans la révolution française de 1848 , la plupart de ses écrits eux-mêmes, plaident un peu bien fort contre les vertus dont vous l'ornez à plaisir. ${ }^{176}$

Cette mise en garde, inefficace parce que trop peu précise, révélait plus de bonne volonté que de compétence; surtout elle ne dissipait pas l'équivoque que l'on entretenait soigneusement sur la religion du poète. Le rédacteur de la Patrie fut heureux de profiter de l'inexpérience de ses censeurs: "Lamartine, me dit-on, n'est pas vraiment religieux. Mais sur quoi se basent mes correspondants pour avancer ce fait? Dois-je leur demander s'ils ont lu Lamartine? Mais s'ils l'ont lu, où donc est le passage qui prouve que Lamartine n'est pas religieux?" Stimulé par la contradiction, le journaliste payait d'audace et écrivait sans sourciller: "Pas religieux, lui! mais il n'est pas tombé de sa plume un seul vers, - que dis-je? un seul mot, - en faveur de l'irréligion ${ }^{177}$, et il la combat, sachez-le, par principe et par conviction"178.

Les étranges propos de Chevalier ne purent impressionner que les badauds. Les lecteurs informés savaient de quel bois se chauffait le personnage. Ils avaient noté, sur le Courrier des États-Unis et sur les journaux canadiens-français, les événements qui l'avaient conduit

176. Patrie, 3 novembre 1856.

177. Par une étrange coīncidence, Lamartine reprenait, justement en novembre 1856, à Saint-Point, dans le neuvième entretien de son Cours familier, l'éloge de Voltaire: "On s'est souvent étonné, depuis que nous pensons tout haut dans ce siècle, de notre admiration continue et persévérante pour ce grand écrivain [ ... ]. C'est que Voltaire est plus qu'un écrivain et plus qu'un poète à nos yeux, c'est une date; c'est la fin du moyen âge. c'est plus encore, c'est la France elle-même incarnée." (Cours familier de litterature, 1: 163-164.)

178. Patrie, 3 novembre 1856. 
au Canada. Ils s'étaient surtout rendu compte de la couleur de ses opinions religieuses et politiques. Proscrit par le coup d'Etat du 2 décembre ${ }^{179}$, Émile Chevalier fit partie du contingent des vingt mille Français qui vinrent se réfugier aux États-Unis en $1851^{180}$, où il collabora au Courrier des États-Unis. Il démissionna le 12 mars 1853, lorsque ce journal devint bonapartiste en fusionnant avec le Phare de New-York ${ }^{181}$, et au lieu de se joindre à ses confrères qui fondaient le Républicain ${ }^{182}$, il vint résider à Montréal, où il fut reçu par nos radicaux avec de grands témoignages d'estime ${ }^{183}$. Avant même de séjourner dans la métropole canadienne, il écrivait dans le Pays. Son premier article ne laissait aucun doute sur sa qualité de révolutionnaire authentique: "Ce que je suis? - Un républicain socialiste. Ce que je veux? Des réformes socialistes. Ce à quoi j'aspire? A l'abolition des nationalités. ${ }^{184 " ~ Q u e l q u e s ~ m o i s ~ p l u s ~ t a r d ~ i l ~ r e m p l a c ̧ a i t ~ l ' u n ~ d e ~ n o s ~ d e ́ m o c r a t e s ~}$ de la plus belle eau, Pierre Blanchet, à la rédaction du Moniteur canadien $^{185}$. Entre temps il avait pris la direction d'une récente publication, la Ruche littéraire ${ }^{186}$, qu'il alimenta de quelques romans indiens imités de Cooper, à la grande satisfaction de François-Xavier Garneau ${ }^{187}$. Sa débordante activité journalistique et littéraire ne l'empêcha pas de participer activement aux discussions de l'Institut Canadien, où même dans ce milieu prêtrophobe, il ne tarda pas à se signaler par ses audaces ${ }^{188}$, et d'y professer un cours de littérature française ${ }^{189}$,

179. Pays, 28 mai 1853; L. Le Jeune, O.M.I. Dictionnaire général du Canada (2 vol., Ottawa, [ 1931 ], 1: 384, s.v. Chevalier.

180. Arthur Charles Cole, The Irrepressible Conflict, 1850-1865 (Coll. A History of American Life, Vol. VII, New-York, [ s.d. ], 128.

181. Courrier des Etats-Unis, 12 mars 1853.

182. Weill, Histoire du parti républicain en France, 292.

183. Pays, 12 avril, 1853.

184. Pays, 3 mars 1853.

185. Moniteur canadien, 13 octobre 1853.

186. Pays, 14 avril 1853.

187. F.-X. Garneau, Voyage en Angleterre et en France dans les années 1831, 1832 et 1839. Extrait publié par le Journal de Québec, 23 novembre 1854.

188. A la séance du 2 novembre 1854 , il proposait comme sujet de discussion: "Le mariage civil serait-il préférable au mariage religieux?" et il s'inscrivait pour l'affirmative. Pays, 7 novembre 1854.

189. Théophile Hudon, s.j., L'Institut Canadien de Montréal et l'Affaire Guibord (Montréal, 1838), 25. 
justifiant dans son cas le mot de la fille d'un exilé: "Un proscrit qui n'enseigne pas devient un proscrit phénomène"190.

Par quelle adroite tactique un publiciste aussi peu orthodoxe s'était-il introduit dans le bureau de rédaction de la Patrie, journal que l'ultramontain Henry de Courcy prisait fort ${ }^{191}$ ? Toujours est-il qu'en novembre 1856, Émile Chevalier s'efforçait, avec un zèle digne d'une meilleure cause, de donner le change sur le déisme de Lamartine: "La religion! mais elle est innée chez $M$. de Lamartine! mais c'est son âme, sa poésie, son souffle. Otez à Lamartine ses instincts, ses croyances religieuses, et il n'est plus rie a"152.

La pétulance du journaliste s'aheurta à un communiqué précis, calme, bien au point, que la Patrie dut insérer dans son numéro du 12 novembre. In s'intitulait: $L a$ vérité avant tout et était signé par Un Canadien français catholique:

Il est évident qu'il y a méprise dans le public catholique au sujet de Lamartine et de ses œuvres. [...]

On veut à toute force et sur tous les tons que Lamartine soit religieux; et dans un pays comme le nôtre, nous dit-on, par compliment, Lamartine, à ce titre large, doit trouver chez les Canadiens sympathie entière. Sa religion comme ses infortunes doivent également nous constituer ses amis dévoués. Halte là! Messieurs ses admirateurs quand même. Passe pour les infortunes: elles méritent tout ce que vous demandez; et nous nous joignons bien cordialement à tous vos nobles sentiments sur ce point. Le génie dans le malheur se recommande par luimême, comme don de Dieu, à toute âme bien née. Mais il faut en rester là avec Lamartine. Autre ch'ose est le génie, autre chose l'usage qu'on en fait. L'un vient de Dieu, il est toujours bon; l'autre est de l'homme et participe à sa nature faible et faillible. Le vrai et le plus grand malheur de Lamartine vient de ce que cette belle intelligence a perdu, ou n'a jamais eu, comme tant d'autres beaux esprits parmi ses compatriotes, la foi de ses pères et des nôtres: la foi catholique. [ ...]

Jocelyn, la Chute d'un Ange, le Voyage en Orient sont des preuves péremptoires des idées étrangement religieuses de Lamartine. Impossible à un catholique éclairé d'y trouver

190. Mme Quinet, Mémoires d'exil, 2: 35. Cité par Weill, op. cit., 286.

191. Lettre de Henry de Courcy à Jacques Viger, 12 septembre 1854. Archives du Séminaire de Québec.

192. Patrie, 3 novembre 1856. 
l'orthodoxie de sa foi. Il y a donc pour le moins à s'étonner de la confiance et de l'aplomb avec lesquels on vient exalter ces œuvres, condamnées, du reste, par l'Église: tribunal sans réplique pour tout vrai catholique.

Tout ce que vous affirmez dans le grand poète constitue surtout pour la jeunesse ou les personnes non assez instruites un danger très grave. La vérité catholique s'y efface sous le clinquant des expressions, ou s'y perd dans le vide, ou s'y embrouille dans le sentimentalisme [...].

Lamartine, dites-vous, est religieux par instinct; son souffle, son âme respire, exhale la religion. Il faut aux catholiques quelque chose de mieux. Il leur faut du défini, du vrai, de l'infaillible, de l'éternel, et Lamartine n'est rien de tout cela. Comète errante, flambeau trompeur, frêle roseau, créature indigente, qui crie aujourd'hui ses misères par le monde et qui ne trouve ses consolations que dans la religion du sort, qu'il accuse en absolvant les hommes, qui sont bons, dit-il: tout cela n'est point fait pour enthousiasmer les vrais catholiques. Il faut bien en prendre son parti, le catholicisme en Canada est encore assez vivant dans les cœurs pour ne pas le confondre avec l'idéologie poétique d'un cœur naturellement tendre et expansif. [ ...]

Donc, en résumé, que le talent de Lamartine reste ce qu'il est; que son infortune soit reconnue et soulagée, nulle contradiction à cet égard. Quant à sa religion instinctive, poettique, illuminée, c'est en Canada que le beau génie de cet homme doit trouver des vœux et des prières pour que ce génie brille de tout l'eclat de sa véritable lumière' ${ }^{193}$.

Ce n'était pas la première fois, au Canada, que l'on se prononçait de la sorte sur l'attitude à adopter envers les conceptions religieuses du poète. Qu'il nous suffise de mentionner quelques faits révélateurs. En 1843, alors qu'un Amédée Papineau consignait dans son Journal, qu'il prenait chez Lamartine, comme chez La Mennais, "l'essence de toutes [ ses] croyances sociales, politiques et religieuses"194, les $M e ́-$ langes religieux exhortaient les jeunes gens à ne lire que le "Lamartine catholique"195. Quatre ans plus tard, le même journal faisait une déclaration plus explicite. La Revue canadienne avait publié le discours de

193. Patrie, 12 novembre 1856.

194. Journal d'Amédée Papineau, 30 octobre 1843. Copie dactylographiée, 5: 172. Archives de la Province de Québec.

195. Melanges religieux, 13 janvier 1843. 
Lamartine prononcé à Mâcon, en juin 1847, en déclarant que cette pièce d'eloquence était "aussi admirable dans le fond que dans la forme"196. Les Mélanges ne furent pas lents à protester. Dès le 31 aout, il paraissait nécessaire au rédacteur "d'entrer dans les plus grands détails pour bien faire comprendre $[. .$.$] ce que la presse catholique$ en France comme ailleurs [ pensait ] de ce discours". Il empruntait un extrait d'un article paru dans l'Ami de la religion, mais comme cette critique ne donnait "qu'une faible idée du discours de M. de Lamartine", le journaliste déclarait qu'il ne voulait pas s'en "tenir là"; il avait donc "recours à un autre journal français: c'est l'Univers qui va nous dire ce que nous devons penser de cette improvisation du grand poète." Puis, ayant reproduit la majeure partie de l'article de Veuillot, il concluait que c'était suffisant pour montrer que le discours de Lamartine renfermait "bien des principes anti-catholiques, bien des idées d'hommes irréligieux" ${ }^{\prime 197}$.

Un Canadien franşais catholique rejoignait donc, en 1856, le publiciste des Mélanges religieux. Au vrai, n'était-ce pas le même homme qui mettait les catholiques en garde contre l'influence du déisme lamartinien? Le rédacteur du journal montréalais, en 1847, était le jeune Hector Langevin ${ }^{198}$. Or, en 1856, à l'Archevêché de Québec, on projetait de l'attacher à la rédaction, avec Joseph-Charles Taché, du Courrier du Canada ${ }^{199}$, qui commença à paraître au début de février $1857^{200}$. Ayant été pressenti et désireux de montrer son savoir-faire, il n'est pas du tout impossible que la mise au point opportune, La vérité avant tout, fât de sa plume.

Mais ces sages considérations n'empêchèrent pas les membres de l'Institut Canadien de favoriser la souscription du poète. Bien plus, elles suscitèrent certes chez eux le désir d'en faire un succès, car n'étaitce pas une occasion toute trouvée de marquer, encore une fois, leur opposition au clergé et aux catholiques? Le Pays du 20 novembre

196. Revue canadienne, 27 aout 1847.

197. Melanges religieux, 31 aout 1847 .

198. Il prenait congé de ses lecteurs en 1849 , après, écrivait-il, deux ans de rédaction. Cf. Mélanges religieux, 20 juillet 1849 . Cf., en outre, Le Jeune, Dictionnaire général du Canada, 2: 61, s.v. Langevin (Hector-Louis).

199. Lettre de l'abbé Bélanger à Mgr Bourget, 2 août 1856. Archives de l'Archevêché de Montréal.

200. De fait il fut l'un des rédacteurs de ce journal. Cf. Le Jeune, op. cit. 
annonçait que l'"entreprise si belle et si désintéressée de M. Desplace [ faisait] des progrès sensibles":

Nous sommes heureux, ajoutait le journaliste, de constater que dans les deux principales villes de notre pays le représentant de M. de Lamartine a été l'objetet d'un accueil distingué autant que flatteur. L'annonce du Cours familier de littérature a trouvé du retentissement jusque dans nos villages les plus reculés. Les journaux de St-Hyacinthe et de Trois-Rivières l'ont publiée dans leurs colonnes, et des lettres que nous recevons de la campagne nous apprennent que la souscription marche rapidement.

Une semaine plus tard, nouveaux témoignages de dévouement au poète: l'Institut Canadien inaugurait une série de causeries sur Lamartine, en recevant officiellement, le 27 novembre, son chargé d'affaires, qui avait quitté Québec la veille ${ }^{201}$. Ce fut tout simplement un triomphe, si du moins l'on en croit le Pays:

A huit heures précises, M. Desplace est monté à la tribune, au bruit des acclamations de la foule, et une heure durant, il a déroule, dans un style digne de l'illustre poète luimême, les divers incidents de la vie littéraire de l'auteur de Jocelyn, des Girondins et de tant de chefs-d'œuvre. Dans la deuxième partie de sa causerie, le conférencier pénétra "plus intimement dans les habitudes de vie et de caractère de Lamartine". Ce discours moitié lu, moitié parlé, fut parsemé des plus heureuses citations tirees des œuvres en prose et en vers du poète mâconnais, et fréquemment interrompu par l'enthousiasmes applaudissements.

Desplace n'eut garde d'omettre de célébrer Madame de Lamartine, qui le méritait bien, d'ailleurs, et de s'attirer, de la sorte, un regain d'ovations:

Les détails donnés par M, Desplace sur Madame de Lamartine qui, comme on le sait, est anglaise, ont été très applaudis, surtout par les compatriotes de cette dame. [...] Mais ce qui la rend plus digne d'admiration, c'est son inépuisable bienfaisance. M. Desplace a été vivement applaudi

201. Journal de Québec, 27 novembre 1856. 
lorsqu'il a parlé de cette école de petites paysannes auxquelles Mme de Lamartine explique de son mieux les premières notions de la religion. ${ }^{202}$

Le lendemain soir, Louis-Antoine Dessaulles, qui n'avait pu prononcer son discours après celui de Desplace à cause de l'heure tardive, entretint ses auditeurs du rôle politique de Lamartine. Il était maître de son sujet, puisque, rédacteur à l'Avenir, il avait pu suivre jour par jour les différentes phases de la Révolution de Février. Mais avant d'aborder l'objet de sa causerie, il tint à réfuter une "correspondance" parue dans la Patrie. Il s'agissait de l'écrit d'un Canadien français catholique. Le démocrate, en butte aux tracasseries des catholiques à cause de ses idées avancées ${ }^{203}$, fut bien aise de prouver "par des citations heureuses du sublime auteur, que jamais écrivain plus religieux, jamais moraliste plus profond n'avait existe"; toujours d'après le journaliste du Pays, "il souleva un véritable enthousiasme dans la salle en rappelant le noble rôle, tout de dévouement et de courage joué par Lamartine dans la révolution de 1848"204.

Un troisième conférencier monta à la tribune de l'Institut Canadien, le 2 décembre, pour clore ce concert de louanges à l'adresse du poète. C'était un Français du nom de Charles-Louis Marle, qui se glorifiait, à tort probablement, d'être né, lui aussi, "à Mâcon, à côté

202. Pays, 29 novembre 1856. - Cette école, Mme de Lamartine l'avait fondée, dès 1823, à Saint-Point. Elle composa pour les enfants qui la fréquentèrent un ouvrage classique: Explications familières des devoirs du dimanche. (Cf. Ethel Harris, Lamartine et le peuple (Paris, 1932). 50) En 1840, elle rédigeait, à la même intention, une Explication familière des principales vérités de la religion à l'usage des jeunes enfants des villes et des campagnes, manuel qui eut plusieurs éditions. (Cf. Luppé, Les Travaux et les jours d'Alphonse de Lamartine, 242.) - Mme M.-Th. Emile Ollivier résume son jugement sur Mme de Lamartine en ces termes: "La noble femme, si digne de vénération pour sa haute vertu, ses malheurs et son inépuisable dévouement" (Valentine de Lamartine (Paris, 1908), 13).

203. Neveu de Louis-Joseph Papineau, son oncle le considérait comme un "autre lui-même". (Louis-Joseph Papineau à John Arthur Roebuck, 11 octobre 1839, Archives de la Province de Québec.) Il serait intéressant de tracer la courbe de l'évolution des idées de Dessaulles, à partir du jour où il jetait l'anathème au La Mennais apostat (Cf. ses Reflexions sur la chute de M. de La Mennais. Extrait cité par l'abbé Joseph-Sabin Raymond, Journal des Trois-Rivières, 15 mars 1867) jusqu'à l'époque où Mgr Bourget le considérait "comme l'ennemi le plus dangereux" qu'avait "la religion dans notre bon pays" (Mandements, lettres pastorales, circulaires et autres documents publiés dans le diocèse de Montréal depuis son érection (Montréal, 1887), 6: 403).

204. Pays. 2 décembre 1856. 
du berceau de M. de Lamartine" 205 , mais était plus véridique quand il se disait "son ancien collègue au conseil municipal de cette ville"206. Sa causerie fut un événement littéraire, si l'on en croit les journaux de l'époque: "Pour nous, écrivait le journaliste de la Patrie, qui avons eu le bonheur d'entendre cet homme vraiment extraordinaire, nous avons été remué par la force irrésistible de son éloquence et nous le saluons "maître de la parole". En effet, M. Marle ne lit pas un discours préparé à grands frais, il ne fatigue pas le public par une lecture monotone, mais il improvise depuis le commencement jusqu'à la fin. Et quelle improvisation! quelle chaleur! quelle animation! quelle mâle énergie!"'207

Ainsi l'Institut Canadien n'avait pas épargné les efforts pour recevoir dignement l'envoyé de Lamartine et, l'esprit de parti aidant, pour activer la diffusion du Cours familier de littérature. Le succès fut-il proportionné à ce déploiement de zèle et d'éloquence? On devait souscrire chez Fabre et Gravel, libraires à Montréal, et à l'Institut Canadien, où S. Martin enregistrait les abonnements ${ }^{208}$. Une lettre de ce dernier à Lamartine, datée du 20 décembre 1856, lui apprenait qu'on en avait recueilli une cinquantaine ${ }^{209}$. C'était tout de même plus honorable qu'aux Etats-Unis!

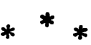

205. Lettre de Marle, 28 novembre 1856, à P.-V.-H. Bourgeau, secrétaire-correspondant de l'Institut Canadien. Pays, 2 décembre 1856. - Monsieur Arthur Morgand, archiviste en chef de Saône-et-Loire et bibliothécaire de l'Académie de Mâcon, veut bien nous écrire que "Ch.-L. Marle ne parait pas être originaire de MAcon, où l'on ne trouve pas trace de sa naissance dans les registres d'état-civil de $\mathbf{1 7 7 0}$ à 1802." - On ne connait pas la date exacte de la naissance de Marle, mais il était directeur, en 1820, de l'école d'enseignement mutuel de Mâcon, Cf. Répertoire des familles notables de Tournus et de sa région (Mâcon, 1915), 249. Renseignement aimsblement communiqué par M. Morgand.

206. Il avait été élu conseiller municipal de Mâcon le 28 mai 1837 et en exerçait encore les fonctions en 1840. Détail transmis par M. Morgand.

207. Patrie, 3 décembre 1856.

208. Pays, 25 octobre 1856.

209. Lettre signalée par Ethel Harris, Lamartine et le peuple, 402, et conservée dans les Archives de Saint-Point. - Le total des abonnements pour les Etats-Unis et le Canada ne dépassait donc pas la centaine. C'était tout simplement dérisoire en comparaison de ce qu'on avait espéré. Aussi Mme de Lamartine écrivait-elle a Charles Alexandre "qu'avec tous les efforts de M. Desplace, rien [ n'était venu ] de l'Amérique du Nord" (Charles Alexandre, Souvenirs sur Lamartine, 349). 
Jean-Baptiste Desplace quitta peu après Montréal et, en passant par la Louisiane, organisa une autre souscription ${ }^{210}$. Au commencement de février 1857, il s'embarquait pour la France ${ }^{211}$, fort dépitéte de l'insuccès quasi total de ses efforts, mais sans doute se rassérénait-il quelque peu lorsqu'il se remémorait les réceptions enthousiastes qu'on lui avait ménagées à Québec et à Montréal. Toutefois, même cette maigre satisfaction d'amour-propre dut tourner à l'aigre, s'il prit connaissance de l'article que Eugène Veuillot consacrait, dans l'U nivers du 5 janvier 1857, à sa mission d'outre-Atlantique.

Les Veuillot, Louis et Eugène, étaient tenus au courant des manifestations intellectuelles et religieuses du Canada par l'un de leurs amis, Henry de Courcy de Laroche-Héron, correspondant de l'Univers à New-York depuis 1845 . Une maladie de poitrine, qui devait le conduire prématurément au tombeau, l'obligea à regagner la France en avril $1856^{213}$. Mais il tint à continuer de suivre la marche des événements au Canada par le Journal de Québec, ${ }^{214}$ qu'il recevait gratuitement, grâce à sa collaboration, depuis $1845^{215}$.

Quoiqu'on n'en ait pas des preuves positives, il ne fait pas de doute que Eugène Veuillot fut, en l'occurrence, inspiré par De Courcy, car les attaques contre nos radicaux étaient trop précises pour avoir été dirigées au hasard: le correspondant de l'Univers avait certes guidé, à Paris, l'arme qui blessait au défaut de la cuirasse.

210. C'est ce qu'annonçait le Pays du 20 novembre 1856. - On se souvient que c'était l'itinéraire tracé par Lamartine lui-même.

211. National, 24 février 1857. - Un billet affectueux de Lamartine l'attendait à Londres: "Soyez le bienvenu dans ce vieux Monde! Vous y retrouverez tous vos vieux amis, et moi surtout, bien reconnaissant d'un tel dévouement. Nous vous attendons impatiemment. [... ] Ici, cela ne va ni bien, ni mal, mais je ne suis ni content. ni sauvé. L'Amérique nous ayant manqué, il faut travailler quatre ans pour se libérer, Dieu nous les donne!" (Charlier, op. cit., 174.)

212. "Desplace ne se consola jamais de n'avoir pas rapporté un million à Lamartine... Il se sentait comme imprégné de toute la honte de l'indifférence américaine... Il se croyait responsable de la petitesse d'un grand peuple. Mais son désespoir ne fit qu'accroître son amitié, et celle qui lui était rendue. Lamartine, en aucune occasion, ne lui laissa voir qu'il avait compté sur une pleine réussite" (Henri de Lacretelle, Lamartine et ses amis, 84).

213. Il fit ce voyage en compagnie de l'abbé Ferland, l'historien. Cf. le Journal de l'abbé Ferland, Archives de l'Archevêché de Québec.

214. Courcy à Mgr Cazeau, 11 juillet 1856. Archives de l'Archevêché de Québec.

215. Courcy à Joseph Cauchon, 29 mars 1854. Collection Bois, Archives du Séminaire de Nicolet. 
Il n'en était pas à sa première polémique contre les libéraux canadiens. Stimulé par Jacques Viger ${ }^{216}$, il les houspilla à maintes reprises dans l'Univers, à la grande satisfaction des journaux conservateurs, comme la Minerve et le Journal de Québec ${ }^{217}$. Il eut surtout la partie belle quand il apprécia le célèbre pamphlet politique publié en 1854, La Pléiade rouge. De Courcy n'avait pas tardé à confier à son correspondant montréalais qu'il trouvait cette brochure "très bien écrite"218. Il ne put résister à la tentation d'en publier un extrait dans l'Univers de décembre 1854, en le faisant précéder de ces lignes: "Nous avons lu, écrivait le journaliste, avec un vif intérêt cette très amusante galerie de portraits contemporains. La vigueur du style, le bon gout de la critique, la bienveillance même de la plaisanterie, donnent à cette œuvre distinguée une place à part dans le journalisme, et il y a plaisir à voir la littérature française cultivée avec tant de talent au Canada. Ces portraits peuvent se lire sans que l'on connaisse les originaux; ils sont si bien tracés qu'ils deviennent des types, et ils nous rappellent à s'y méprendre telles et telles de nos illustrations de 1848, qui ne parvinrent aussi à escalader la tribune que pour se précipiter dans le ridicule ou l'oubli public." 219

La mission de Desplace fournissait à De Courcy une nouvelle occasion de s'en prendre au radicalisme canadien-français, qu'il appelait "la plaie honteuse de la colonie"220. Il la saisit aux cheveux en suggérant à Eugène Veuillot de commettre, à leur endroit, quelques lignes de sa manière. Il convient de citer de larges extraits de cet article, qui provoqua une explosion de colère dans le eamp libéral:

Depuis six mois, M. de Lamartine a entrepris, en Amérique, une campagne qui paraît devoir être fort avantageuse à ses finances. On quête pour lui au Brésil ${ }^{221}$, au Canada, aux

216. De Courcy écrivait à Viger, le 1er novembre 1854: "Vous me dites que je devrais tonner contre vos rouges. (Archives du Séminaire de Québec).

217. Il serait trop long, pour notre propos, d'entrer dans le détail de ces polémiques.

218. Courcy à Viger, 17 novembre 1854 .

219. Univers, 30 décembre 1854.

220. C[ ourcy ] de Laroche-Héron, Les Servantes de Dieu en Canada (Montréal, 1855), 141.

221. Le Brésil souscrivit généreusement au Cours familier. Cf. Mario de LimaBarbosa, Lamartine et le Brésil (Paris, 1927). 
États-Unis. Le grand poète a expédié vers le Nouveau-Monde un de ses admirateurs, M. Desplace, lequel a réussi à former un comité composé des plus grands noms politiques et littéraires des États-Unis. Ces messieurs ont flattés de recevoir de Paris des billets path6tiques, où $M$. de Lamartine leur parle de son cœur qui saigne, de ses entrailles qui s'arrachent, de sa poitrine brisée, de son sein meurtri, et autres métaphores. On a fait aussitôt un appel eloquent au peuple américain, pour le presser de secourir la grande infortune lui offrant en échange le Cours familier de littérature.

Tant que la souscription ne sortait pas des limites des États-Unis, nous n'avions rien à en dire, et nous étions charmés, au contraire, que les Américains, qui sont riches, vinssent apporter leurs dollars au bureau d'Apollon. Mais voilà que l'on se prépare à traire aussi l'Amérique du Sud et le Canada. Le diligent M. Desplace est aujourd'hui à Montréal, organisant un comité pour débiter le Cours familier de littérature. Ce comité a fait un appel au peuple, [...]

Cet appel, reproduit par tous les journaux, est signe des chefs du parti rouge et socialiste du Canada. Rien de mieux. Mais nous y voyons aussi figurer les noms les plus considérables du parti français catholique, et nous en sommes surpris. Venir vanter les sentiments religieux du poète, et exalter le bien que ses écrits ont fait à la France, c'est se montrer un peu trop en retard sur l'histoire. Les Canadiens sont encore sous l'impression des Méditations et des Harmonies poétiques; mais, depuis lors, il y a eu la Chute d'un Ange, les Girondins et le reste; il y y a eu la glorification de Robespierre, les Confidences intimes, où le matérialisme nous a semblé plus cultivé que le spiritualisme; il y a eu les romans, dont un : mère fera bien de ne pas permettre la lecture à sa fille. En honorant de leur patronage les œuvres de M. de Lamartine, les Canadiens oatholiques nous semblent donc trop prouver qu'ils ne les ont pas lus, et ils nous pardonneront d'avoir cherché à les éclairer. [ ... ]

Les Canadiens paraissent, en effet, très décidés à prendre au sérieux la mission de M. Desplace; et non contents de remplir l'escarcelle du frère quêteur, ils ont voulu remplir son estomac. Un banquet national lui a été offert; on a entouré des plus grands honneurs l'ami de l'illustre poète. [...] ]

M. de Lamartine se doit à lui-même de signifier aux naîfs souscripteurs du Canada, de Bahia et autres lieux, qu'il n'est plus l'homme de ses premières poésies. Ces braves gens en sont restés là, et, en souscrivant pour le philosophe humanitaire, l'apologiste de la révolution, l'admirateur de l'Islamismo; ils croient venir en aide au poète religieux. Il faut les avertir 
de la móprise où ils tombent, et leur déclarer que si leurs offrandes ne se trompent pas de nom, elles se trompent de but. Si M. de Lamartine ne veut pas faire cela, s'il accepte des souscriptions motivées d'une façon fort blessante pour son present, qu'il reconnaisse au moins les bons sentiments de ces admirateurs attardés, en ne leur envoyant pas son Cours familier de littérature ${ }^{222}$.

Cet article fut reproduit par le Journal de Québec du 31 janvier 1857. Le rédacteur, Émile de Fenouillet, qui, ayant cédé à l'enthousiasme du moment, avait célébré Lamartine, se sentait, lui aussi, atteint par les lignes mordantes de Veuillot ${ }^{223}$. Il essaya de riposter, mais en réalité il éludait la question: en mettant l'accent sur l'affection des Canadiens français pour la France et sur la compassion que devait susciter une immense infortune, il s'écartait très habilement du point névralgique du débat: la religion de Lamartine ${ }^{224}$.

Ce n'est donc pas de Fenouillet, l'ancien collaborateur de l'Univers, que pouvait naturellement venir une fin de non-recevoir absolue a l'article de Eugène Veuillot, mais de ceux qui étaient directement mis en cause: les libéraux-radicaux. Les démocrates, encore une fois, étaient vertement pris à partie, et c'était un Veuillot qui venait à la rescousse du parti conservateur, du "parti catholique", suivant la terminologie partisane de la Minerve: aubaine doublement appréciable! Comme bien l'on pense, les libéraux ne furent pas d'humeur à se tenir cois. Le National, journal fondé à Québec en 1855, pour soutenir dans la région les intérêts démocratiques, se chargea de répondre à Eugène Veuillot. Il le fit en une prose ridicule, mais dont il faut citer quelques paragraphes, ne serait-ce qu'à titre de curiosité historique:

...nous ne pensions pas que le fanatisme de cette feuille [ l'Univers] avait les bras assez longs pour nous atteindre jusque sur ce coin de terre de l'Amérique et tendrait à nous

222. Univers, 5 janvier 1857.

223. De Courcy avait sans doute sursauté en lisant, dans le Journal de Québec, les éloges de Fenouillet à l'endroit de Lamartine. Il n'aimait pas, d'ailleurs, le journaliste: “...je ne comprends pas, écrivait-il à Viger, le 6 aoât 1855, le goât des Québecquois de supporter une pareille huitre." (Archives du Séminaire de Québec)

224. L'article de Fenouillet fut inséré dans la Patrie, 4 février 1857. M. Marion en a cité des extraits dans son travail, "Lamartine et l'Institut Canadien de Montréal", Revue de l'Université d'Ottawa, 20 (janvier-mars 1850): 39-42. On pourra s'y référer et étudier en même temps un échantillon caractéristique de la manière de M. Marion, nous voulons dire les commentaires dont il agrémente ces citations. 
entourer de ses sinistres lueurs; nous ne pensions encore moins que ce Français qui porte le nom de Veuillot viendrait, en sa qualité de catholique, afficher son ignorance de nos affaires et accuser de niaiserie les sentiments respectables qui poussent l'élite d'un pays à rendre service à un homme de génie, à insulter à toute une nationalité unie de cœur à la France et à ses gloires, parce qu'elle rend hommage à un de ses enfants les plus nobles, à celui qui, par sa grandeur d'âme inaltérable et son talent, honore le plus l'humanité et dont la tête incline aujourd'hui vers la tombe sous le triple poids de l'âge, du sacrifice et de la pauvreté.

Nous ne voudrions pas nous attaquer a ce journal d'impurs prédicants [...]. Nous ne nous en occuperions pas, si l'article dont nous parlons ne contenait des erreurs a notre sujet qui nous font paraitre sous un faux jour comme parti, et ne proviennent que des mauvais renseignements et des calomnies des journaux de ce pays contre une partie de notre population. [ ...]

Mais que peuvent nous faire à nous, nous nous le demandons, hommes libres et dédaigneux de ces interventions étrangères, les articles haineux et insultants de cette feuille qui entend parler, à l'instar du Souverain Pontife, urbi et orbi, à la ville et au monde, bénir et foudroyer, de même sauver et maudire au nom du Christ. Halte-là, Tartuffe! Entre vous et nous, il y a la mer, la mer puissante et saine qui nous sépare de vos fanatismes et de vos cyniques brocantages et qui nous permet d'adorer Dieu dans la simplicité de notre cœur, à l'abri de vos passions, de tendre la main a toutes les infortunes et à tous les naufrages. [...]

Il est une partie de cet article que nos lecteurs ont dû voir entre autres, c'est celle où l'on désigne les partis qui nous divisent. Il y a, suivant l'Univers, un parti rouge et socialiste et un parti catholique!?

D'où lui vient cette opinion erronée, sinon de ces journaux de notre pays, qui nous font connaitre toujours, par des calomnies méprisables, comme des rouges, des socialistes, des destructeurs du trône et de l'autel. Tels sont les résultats que produit et produira toujours cette presse qui ne vit que de prejugés et de l'ignorance d'autrui. Croit-on que cela puisse produire quelque bien parmi nous, lorsque l'on aura imprimé dans l'opinion de personnes étrangères à notre pays l'idée que nous avons renié la croyance religieuse de nos pères, la croyance nationale?

Si l'Univers avait raison en parlant des partis qui existent ici, il se trouverait que la moitié des hommes de la race fran- 
çaise, à peu près, serait anti-catholique! Encore une fois, quel bien peut-il résulter de tout cela? Il faudrait donc, devra-t-on croire naturellement, que les pasteurs qui ont eu charge d'âmes ici et qui reçurent le dépôt de notre foi, aient été de très maladroits administrateurs pour ne pas avoir conservé intacte la foi de leur troupeau dans le Dieu de leurs pères.

Nous n'insisterons pas là-dessus; c'est à la conscience publique à faire justice de ces odieux renseignements et de ces imbeciles erreurs. Nous dirons seulement à l'Univers que, parmi les Canadiens français, il n'y a pas de parti socialiste et de parti catholique. $\Pi$ y a seulement des démocrates et des ministériels pour le quart d'heure, tous bons catholiques généralement. La seule différence qui puisse exister entre ces deux partis, sous le point de vue religieux, c'est que les ministériels ou les tories se servent assez, beaucoup même, de la religion comme d'un moyen pour parvenir à leur but, et que les démocrates respectent trop profondément Dieu et le culte qu'ils lui rendent pour oser agir comme leurs adversaires dans les luttes existantes.

Il n'y a donc, encore une fois, sous ce rapport, que des conservateurs qui vivent par la religion et des démocrates qui vivent pour elle. C'est la seule et importante différence entre eux.

L'Univers pourra voir par là les chances de succès qu'il peut avoir ici et s'il croit pouvoir ouvrir boutique à l'ombre de nos autels.

Nous avons meilleure opinion de nos compatriotes que lui, s'il est d'opinion qu'il réussira parmi nous. Le ciel du Nouveau-Monde n'est pas le ciel de M. Veuillot, ou nous noue trompons fort ${ }^{225}$.

C'est par cette diatribe passionnément partisane que se terminait, sans grandeur, au Canada, la souscription à la publication de Lamartine. Le courant de sympathie qu'avait d'abord provoqué l'appel du poète impécunieux, s'était allé perdre dans le sable de l'indifférence ou dans les marécages de l'invective. Le grand homme, qui déclarait noblement:

225. National, 17 février 1857. - Le journaliste libéral se trompait, en effet, car les conservateurs venaient de fonder (1er février 1857), à Québec, le Courrier du Canada, qui durant toute sa carrière (il devait cesser de paraitre en 1901), serait avec, plus tard, la Vérité de Tardivel, le journal veuillotiste par excellence, surtout sous la direction des Taché, des Dionne et des Chapais. Le National ne tarda pas à discerner la principale influence que subissait le Courrier. Dès le 20 juin 1857, il lui reprochait de "copier à l'instar d'un singe les allures méprisables du journal l'Univers". 
"En fait de haine, je veux mourir insolvable" nôtres un signe de contradiction. Ce n'était, hélas! que la réplique de sa situation en France: l'opposition des catholiques persistera à l'accompagner jusqu'à la tombe exclusivement. Voyons brièvement dans quelles circonstances.

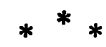

Eugène Veuillot s'était attribue, en janvier 1857, la critique de la souscription lamartinienne en Amérique. Son frère Louis - quoniam nominor leo! - se réservait, pour l'année suivante, la France, l'Europe. Il publiait, le 1er janvier 1858, dans l'Univers, son "célèbre article"227: Du mercantilisme littéraire ${ }^{228}$. Le publiciste s'en prenait aux écrivains qui "rassemblent leurs vieux papiers, [...) content leurs jeunes romans, (...) vendent le secret de leurs amis morts et un peu ceux de leurs amis qui vivent"'229, à Lamartine surtout, qui s'était signalé "entre les illustres qui ont donné le détestable exemple de l'amour du bruit pour l'amour de l'argent": "Où ce licol, accentuait-il, ne l'a-t-il pas traîné? Que de feuillets entassés malgré Minerve, et que de négoces pour placer ces inexorables paraphrases! Il a promené partout sa grasse pauvreté, tendu sa sébile au coin de tous les feuilletons, employé toutes les ruses pour obtenir par cette industrie de la mendicité poétique dont il est le créateur, tout l'or et tout le billon que la compassion et la vanité se peuvent laisser traire." 230

Veuillot n'était pas revenu de lui-même au poète. Béranger avait fourni aux deux hommes un terrain commun où se heurter derechef.

Le journaliste, qu'offusquait à bon droit la réputation usurpée du "chansonnier national", tenait à dégonfler cette immense bau-

226. Cité par Camille Latreille, Lamartine, poète politique, 115.

227. Guillemin, Le Jocelyn de Lamartine, 393. - Par contre, dans un ouvrage postérieur du même auteur: Connaissance de Lamartine, 284, ce n'est plus qu'un "haineux article"!

228. Le journaliste rejoignait les appréciations de Sainte-Beuve sur la "litterature industrielle". Cf. lettre de Sainte-Beuve a Juste Olivier, 2 novembre 1844. Correspondance générale de Sainte-Beuve, 5, deuxième partie: 702-704.

229. Mélanges (T. XXXIII des Oeuvres complètes, Paris, 1935), 7: 299.

230. Ibid., 300. 
drucbe. Par deux fois, en $1853^{231}$ et en $1855^{232}$, il avait dû se désister devant l'opposition du Bureau de la Presse, truchement de la volonté impériale. Mais il crut, en 1857, pouvoir braver le déplaisir de Napoléon III et aller jusqu'au bout de ses convictions, à l'occasion de la mort de Béranger, d'autant plus que Lamartine avait fait de cette mort une apothéose outrée ${ }^{233}$. Il profita de la circonstance pour rappeler sévèrement le chantre des Méditations au respect de lui-même et de son passé catholique ${ }^{234}$. L'auteur du Cours familier de littérature lui accusa réception de ses critiques dans une Lettre [en vers] a $\mathrm{Al}$ phonse Karr, jardinier ${ }^{235}$. Rapprochant le destin de son ami et le sien de celui de Cicéron, le poète écrivait:

Notre Fulvie, à nous, c'est quelque amer Fréron

Dont la haine terrestre au feu du ciel s'allume

Et qui nous percera la langue avec sa plume!

Louis Veuillot citait ces vers dans son article du ler janvier et s'écriait: "Je crois que j'ai tout à fait le droit de prendre ici la parole. Je la prends, et je proteste pour Fréron." La distinction que le polémiste avait si souvent marquée entre les deux manières de l'écrivain, l'allusion historique lui permettait de la rajeunir et de la graver en un relief puissant: "Je le connais très bien, ce pauvre Fréron. Jamais il n'a trouvé que $M$. Karr eût une langue; il n'a donc jamais voulu la percer. Quant à M. de Lamartine, c'est autre chose; il a deux langues:

231. Cf. E. Veuillot, Louis Veuillot, 3: 170.

232. De Courcy communiquait, le 4 juillet 1855, ce renseignement à Viger: "Vous avez peut-être vu dans l'Univers des articles superbes de Veuillot contre Béranger, et remarqué la manière volontairement maladroite avec laquelle Veuillot s'est tu tout à coup, laissant le Siècle chanter victoire. C'est que la police a prévenu l'Univers qu'il fallait respecter Béranger, considéré comme digne d'éloges dans le monde impérial pour avoir contribué à populariser le premier empereur et son petit chapeau. De plus, le ministre de l'Intérieur, Billault, est grand ami du Siècle et ne veut pas que l'Univers maltraite trop cet infâme journal. Vous voyez que le gouvernement actuel, qui se pose en protecteur de la religion, ne le fait souvent que par politique, ce qui rend la sécurité de l'Église bien précaire en France." (Archives du Séminaire de Québec)

233. Entretiens XXI et XXII du Cours familier de litterature. Citations par Veuillot, Melanges, 7: 218-220.

234. Ibid., 216-229. 111-112.

235. Analyse et citations par Camille Latreille, Lamartine, poète politique, 
l'une belle et noble, qui a récité de beaux et nobles vers; l'autre diffuse et chargée, qui a débité en prose et en vers de détestables sornettes, et aussi misérablement flatté l'erreur populaire qu'aucun rimeur de cour les folies d'aucun roi. De ces deux langues, le Fréron que je connais a toujours admiré la première; de grand cœur il percerait (avec sa plume) la seconde. Où est le mal de percer ainsi une méchante langue? C'est à la plume de percer les langues, et souvent elle n'a pas de plus utile emploj."236

Cet article souleva une immense clameur réprobatrice dans le camp des ennemis de Veuillot. Une vingtaine de journalistes parisiens trempèrent dans l'écritoire une plume irritée pour répondre à l'implacable censeur. Au jugement du polémiste lui-même, ce fut "un massacre" ${ }^{237}$. Mais, dédaignant la tourbe des minores, il ne répliqua qu'au seul Brémond. C'était le pseudonyme d'Arthur de la Guéronnière, ancien collaborateur au journal de Lamartine, le Bien public ${ }^{238}$, alors conseiller d'État, chef officiel "des services de l'imprimerie, de la librairie et de la presse ${ }^{239 "}$. La qualité du personnage justifiait l'attention préférentielle du publiciste. Vite débusqué de son incognito, au grand amusement de la galerie, qui s'étendait des plus infimes grimauds à l'Empereur lui-même, La Guéronnière dut demander grâce au terrible jouteur ${ }^{240}$. Mais il se vengea, deux ans plus tard, en se servant de son influence administrative pour faire supprimer l'Univers: le fonctionnaire venait au secours de Trissotin!241

Lamartine, à la rescousse de qui tant d'amis s'étaient levés inutilement, se plaignit à son tour de Veuillot. Dans le vingt-cinquième Entretien du Cours familier, qui débutait par un Préambule de l'année 1858 , l'écrivain s'ouvrait à ses lecteurs “de ce déchaînement d'outrages et de cette recrudescence de colères, que rien ne justifiait, ni dans son passé d'homme politique, pur de toute persécution des personnes ou des idées, ni dans son présent d'écrivain, entièrement étran-

236. Mélanges, 7: 302.

237. E. Veuillot, op. cit., 174.

238. Univers, 28 janvier 1858.

239. E. Veuillot, op. cit., 175. Veuillot.

240. On peut lire dans les Mélanges, 7: 348-381, les spirituelles turlupinades de

241. Cf. E. Veuillot, op. cit. 179. 
ger aux querelles du temps: "D'où viennent donc ces représailles sans griefs, sans justice et sans générosité?", se demandait-il douloureusement. "Hélas! faut-il le dire à la honte de notre espèce? Ce n'est pas parce que nous sommes coupables, c'est parce que nous sommes malheureux!..."242

Le journaliste fut, cette fois, apitoyé par cet accent qui ne trompait pas: il promit de cesser ses critiques ${ }^{243}$. Mais il crut qu'il devait fournir à ses lecteurs une ultime explication de son attitude envers l'immense douleur qu'il avait si souvent rudoyée:

Nous croyons que le travail de M. de Lamartine, depuis douze ou quinze ans, produit des résultats funestes. Tant d'écrits profondément mauvais sous un vernis de décence, profondément sceptiques sous une enveloppe de fade religiosité, profondément révolutionnaires sous les dorures du langage, ne sont pas impunément lus même des esprits sérieux; ils blessent sans remède ceux qu'ils séduisent. Tels sont nos griefs contre M. de Lamartine, tels sont ses crimes, tel est son malheur. Ces griefs sont réels, ces crimes doivent être dénoncés; ce malheur, nous le plaignons, mais notre conscience le reconnaît mérité. S'il engendre d'innombrables angoisses, puissent-elles assez percer le triste cour qu'elles envahissent pour y faire entrer la lumière qui seule les dissipera.

M. de Lamartine est digne de compassion. Il doit souffrir beaucoup pour laisser si souvent échapper ce cri dont il émeut la terre: Je suis malheurenx. Même en faisant la part de la littérature, qui ne perd jamais ses droits, des embarras d'argent, quelle qu'en soitl'étendue, ne sauraient à ce point abattre une âme d'ailleurs courageuse. C'est la situation morale, et non la situation matérielle, qui se lamente avec cet oubli des fiertés ordinaires. Or, une telle plaie n'est pas de celles que l'on peut laver dans le Pactole. Il y faut l'eau et le sang qui coulent du flane de Jésus-Christ. M. de Lamartine ne connaît pas le vrai prix auquel il peut se racheter. Dieu est le créancier qui le tourmente ${ }^{244}$.

Louis Veuillot tint fidèlement sa promesse: l'Univers ne relança plus le vieil écrivain dans sa solitude laborieuse et attristée. Ce n'est

242. Latreille, op. cit, 114.

243. Melanges, 7: 391.

244. Ibid., 392-393. 
qu'à la mort de Lamartine que le journaliste, qui crut, en s'abusant peut-être ${ }^{245}$, à sa conversion, eut enfin à son adresse des paroles d'indulgence: "Vieux, humilié, infirme, écrivait-il le 9 mars 1869, et le pied sur le seuil de cette antichambre de la mort, où il devait rester si longtemps et si loin de sa gloire humaine, il s'est enfin souvenu, il s'est reconnu et, par une grâce longtemps refusée peut-être, il a tiré son âme du naufrage de toutes ses splendeurs."246

C'est par ces lignes graves qui se terminaient sur une note d'espérance que Louis Veuillot tint à clore le douloureux débat qui, depuis tant d'années, l'opposait à Lamartine. Elle serviront également d'épilogue à cette trop longue étude.

Frère Robert SyL vaIN, É.C.

245. Un "lamartinien" aussi averti que $M$. Guillemin penche pour la non-conversion de l'auteur de Jocelyn; il s'est expliqué à ce propos dans plusieurs de ses ouvrages. Nous y renvoyons le lecteur.

246. Mélanges (T. XXXVI des Oeuvres complètes, Paris, 1937), 10: 177. 